\title{
Fragility fracture of the fourth rib in a patient with a cardiac pacemaker: an uncommon case
}

\author{
Ebru Köseoğlu Tohma' ${ }^{10} \cdot$ Özden Özyemişçi Taşkıran² ${ }^{2}$
}

Received: 25 July 2021 / Accepted: 30 August 2021 / Published online: 9 September 2021

(c) International Osteoporosis Foundation and National Osteoporosis Foundation 2021

\begin{abstract}
Purpose In patients with a cardiac pacemaker, pocket-related complications such as nerve impairment or bone fractures are infrequent. We present a man with a fracture of the 4th rib several months after pacemaker implantation.

Case presentation A 74-year-old man, with a left prepectoral pacemaker implanted 13 months ago, presented complaining of chest pain. The pain started after a sudden trunk rotation and right arm flexion movement with a crack. There was tenderness to palpation and crepitation over the left upper ribs. Computed tomography identified a non-displaced fracture line in the anterior aspect of the left 4th rib. After kinesiotaping and activity restriction, pain alleviated.

Conclusion Pacemaker implantation might have caused shoulder dysfunction and pectoral tightness resulting in reduced flexibility of the trunk. Consequently, a reaching motion of the arm with a trunk rotation might have directed rotational force vectors towards the osteopenic left 4 th rib causing a fragility fracture. In elderly with a pacemaker, osteopenia and concomitant sarcopenia may create a predisposition to this atypical complication.
\end{abstract}

Keywords Rib fracture $\cdot$ Cardiac pacemaker $\cdot$ Osteoporosis $\cdot$ Osteopenia $\cdot$ Fragility fracture

\section{Background}

In patients with a cardiac pacemaker $(\mathrm{CP})$, pocket-related complications generally comprise hematoma, skin lesions, infection, device migration, or Twiddler's syndrome (twisting of the pulse generator inside the pocket). Other complications, such as nerve impairment or bone lesions, are extremely infrequent [1]. Low bone mineral density (BMD) was found significantly associated with a higher rate of rib fractures after blunt thoracic trauma in patients 50 years and older [2]. In addition, there are case series of non-traumatic rib fractures following a severe cough or sneeze, not only in the elderly but also in young and middle-aged patients with one common pre-existing condition; osteoporosis [2,

This case report was written according to the CARE checklist.

Ebru Köseoğlu Tohma

ebru_koseoglu@hotmail.com

1 Department of Physical Medicine and Rehabilitation, Muğla Sıtkı Koçman University Research and Training Hospital, Muğla, Turkey

2 Department of Physical Medicine and Rehabilitation, Koç University School of Medicine, İstanbul, Turkey
3]. Though rib fractures are the most common fractures in men over 65 years, a rib fracture caused by a pacemaker has never been reported in elderly [2]. We present an uncommon case of 4th rib fracture in an elderly male with a permanent pacemaker.

\section{Case presentation}

A 74-year-old man, with a left prepectoral permanent pacemaker implanted 13 months ago, was admitted to physical medicine and rehabilitation outpatient clinic complaining of left-sided chest pain. He stated that chest pain (VAS: 6/10) at the anterior-upper costal site started 10 days before his admission, following a sudden trunk rotation to the left side with right arm flexion. He heard a crack when he tried to reach his phone while sitting. The pain was aggravated by twisting movements of the trunk and relieved by rest. He had coronary bypass surgery 9 years ago and aortic valve replacement 6 years ago. He was diagnosed with interstitial lung disease 6 years ago and he required long-term oxygen therapy for the last year. He was hospitalized due to COVID-19 pneumonia with vigorous coughing 9 months ago. He had no history of osteoporosis, bone fractures, 
steroid use, inflammatory arthritis, smoking, substance or alcohol abuse, malignancies, or radiotherapy. He was on carvedilol, furosemide, erdosteine, digoxin, rivaroxaban, hydrochlorothiazide, spironolactone, sacubitril, valsartan, tiotropium bromide, vilanterol-fluticasone furoate, sertraline, and lorazepam.

Body mass index was $23.4 \mathrm{~kg} / \mathrm{m}^{2}$. On physical examination, there was tenderness to palpation with an apparent crepitation anteriorly at the level of the left 4 th rib. He had an increased thoracic kyphosis. Left shoulder motion was restricted at the end ranges due to pain. Posteroanterior and lateral chest $\mathrm{x}$-ray did not help to visualize cortical borders of the ribs below the $2^{\text {nd }}$ (Fig. 1). The dorsal spine showed increased radiolucency of vertebral bodies and radiodensity of the cortical rim, with mild anterior height loss of thoracic vertebrae. Chest computed tomography (CT) identified a non-displaced fracture of the anterior aspect of the left 4 th rib (Fig. 2). BMD values and T scores were $0.901 \mathrm{~g} / \mathrm{cm}^{2}$ and -1.7 in lumbar vertebrae, $0.821 \mathrm{~g} / \mathrm{cm}^{2}$ and -0.8 in the left femoral neck. The serum level of 25-hydroxy vitamin D was $27.2 \mathrm{ng} / \mathrm{mL}$ with a mildly increased serum level of parathyroid hormone $(72 \mathrm{ng} / \mathrm{L})$. Other laboratory test results were normal, including serum calcium level.

He was recommended to avoid aggravating activities such as twisting of the trunk. No nonsteroidal anti-inflammatory drugs (NSAID) were initiated not to worsen cardiac and respiratory status. He was prescribed one dose of $50000 \mathrm{IU}$ oral vitamin D supplementation. Kinesiotape was applied for pain management as I-bands over the $3^{\text {rd }}$ through $5^{\text {th }}$ ribs and intercostal muscles anteriorly and posteriorly with

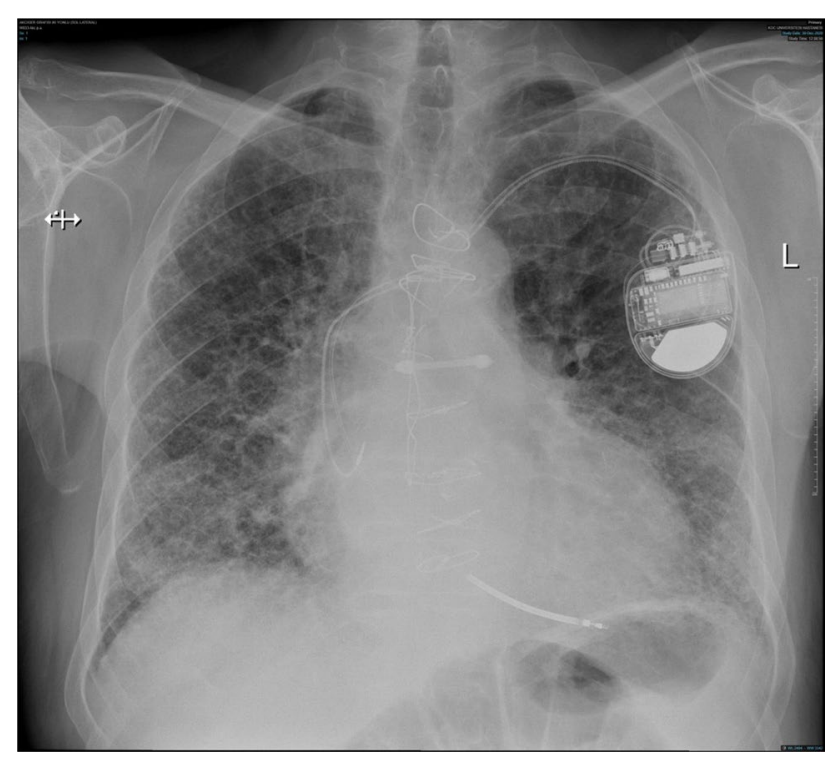

Fig. 1 Posteroanterior chest $\mathrm{x}$-ray is negative for any fractures. The positions of the pulse generator and leads are appropriate with findings of interstitial lung disease and cardiomegaly

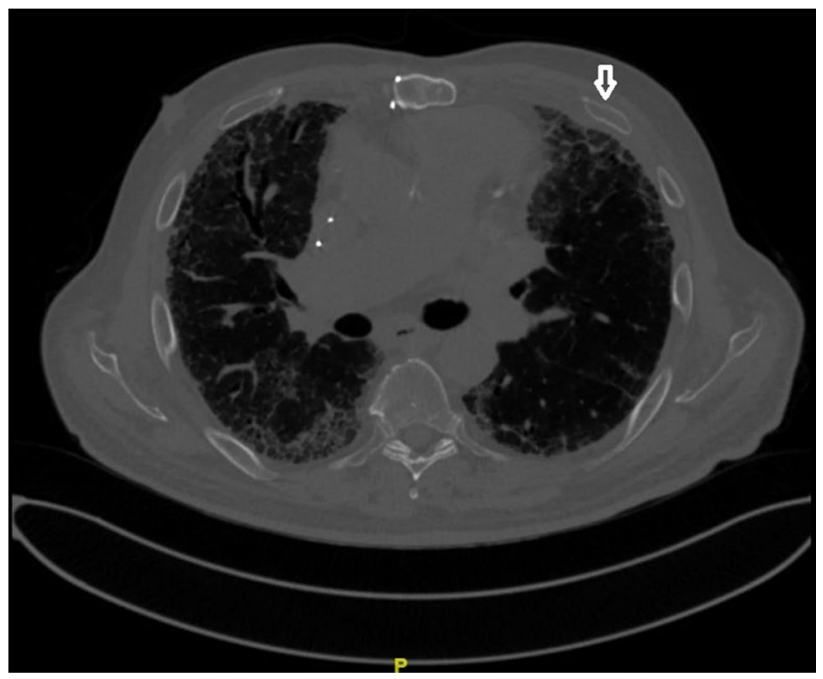

Fig. 2 CT showing non-displaced fracture line in the anterior aspect of the left 4th rib (white arrow)

inhibition technique to stabilize the ribs. Within two weeks pain symptoms alleviated, and the patient described no tenderness to palpation in the fracture zone with painless mobility of the trunk and both upper extremities. Oral alendronate weekly and calcium and vitamin D daily were initiated for osteoporosis management 4 weeks after pain relief. Written informed consent was obtained from the patient for publication of this case report.

\section{Discussion}

Complications involving deeper anatomical structures around the pulse generator, such as brachial plexus or sympathetic nerve fiber injury, muscle impairment, or bone fractures, are rare following a CP implementation [1]. To our knowledge, our case is the second reported rib fracture associated with a CP device. The first case was an asthenic 20 -year-old female with no comorbidities including osteopenia or osteoporosis. In that case, fracture of the $2^{\text {nd }}$ rib was considered a fatigue stress fracture following a daily activity of the upper extremity and assumed to be associated with short subclavicular distance [1]. The onset of her pain was insidious, starting as a discomfort only after 2 months of pacemaker implantation and progressively increasing to severe pain [1]. Contrary to this patient, our patient had a sudden onset of pain and was an elderly male with a normal BMI.

Almost $40 \%$ of rib fractures in older patients are non-traumatic, e.g., after coughing [4]. Our patient had no history of trauma either. In addition, non-traumatic first rib fractures have been reported in young athletes who generally participate in sports with heading or overhead arm movements; 
stress fractures likely to result from the opposite force of muscle contractions on the bones with ongoing maturation [5]. There are case series of non-traumatic rib fractures after coughing not only in elderly but also in young patients, all of whom demonstrated a T-score below - 2.5 [3]. Our patient had osteopenia as well, which we believe was the main predisposing factor for the rib fracture. He also had a history of vigorous and long-term coughing, but his incident rib fracture did not occur following a cough. However, recurrent contraction due to long-term coughing may have caused muscle fatigue in inspiratory and expiratory muscles which may have avoided dissipation of forces and contributed to the fracture in this elderly case with probable sarcopenia[6].

Shoulder dysfunction due to pain or fear of dislocation is reported following CP implantation [7]. In our patient, $\mathrm{CP}$ implantation might have caused disuse of the left upper extremity, resulting in weakness of rotator cuff and shoulder girdle muscles including serratus anterior. Moreover, our patient had pectoral muscle tightness due to pocket formation and kyphotic posture. Weakness of serratus anterior muscle and tightness of pectoral muscles altered the position of scapula anteriorly, contributing to decreased trunk flexibility. He also had sarcopenia due to his advanced age and co-morbidities. All mentioned above might have resulted in inadequate dissipation of rotational force vectors created by daily movements of the upper extremity and persistent coughing. Vectors caused by a sudden movement of ipsilateral trunk rotation with right arm flexion might have forced left-sided pulse generator towards the osteopenic 4th rib, hence fragility fracture.

Radiographs, the first step of diagnosis, are negative in up to $60 \%$ of the patients with a rib fracture [6], such as our patient whose CT scan revealed the fracture of the anterior aspect of the 4th rib (Figs. 1 and 2). NSAIDs, the most widely used analgesics for pain management of rib fractures, are usually relatively contraindicated in elderly. Therefore, we applied kinesiotape, a drug-free, non-invasive treatment which alleviated the pain within 2 weeks. Combining NSAID therapy and kinesiotaping was found to be more effective than NSAIDs alone in pain relief of rib fractures [8].

\section{Conclusion}

In conclusion, we present an osteopenic elderly male patient who is, to our knowledge, the second case of a rib fracture associated with a CP. In this case with reduced trunk flexibility, an everyday motion of the right arm with a trunk rotation to the left side might have directed rotational force vectors towards the osteopenic 4th left rib via pulse generator, resulting in a fragility fracture. Rib fractures, which are probably underdiagnosed due to vague manifestation, must be kept in mind in older patients with chest pain, even there is no history of trauma. In elderly with a pacemaker, low bone mass and concomitant sarcopenia may cause a predisposition to this unexpected complication.

Author contribution All authors contributed to the manuscript. Data collection was performed by Özden Özyemişçi Taşkıran. The first draft of the manuscript was written by Ebru Köseoğlu Tohma and all authors commented on previous versions of the manuscript. All authors read and approved the final manuscript.

Data availability All data and materials support our published claims and comply with field standards.

Code availability Not applicable.

\section{Declarations}

Ethics approval Written informed consent was obtained from the patient for publication of this case report. All the procedures were performed according to the World Medical Association Declaration of Helsinki.

Consent for publication All authors have approved the manuscript and agree with submission to Archives of Osteoporosis.

Conflicts of interest None.

\section{References}

1. Ksela J, Racman M, Zbacnik R et al (2020) Pacemaker-generated stress fracture of the second rib: a case report. J Cardiothorac Surg 15:258. https://doi.org/10.1186/s13019-020-01303-y

2. Prins JTH, Van Lieshout EMM, Reijnders MRL et al (2020) Rib fractures after blunt thoracic trauma in patients with normal versus diminished bone mineral density: a retrospective cohort study. Osteoporos Int 31:225-231. https://doi.org/10.1007/ s00198-019-05219-9

3. Katrancioglu O, Akkas Y, Arslan S, Sahin E (2015) Spontaneous rib fractures. Asian Cardiovasc Thorac Ann 23:701-703. https://doi. org/10.1177/0218492315586485

4. Wuermser L-A, Achenbach SJ, Amin S et al (2011) What accounts for rib fractures in older adults? J Osteoporos 2011:1-6. https://doi. org/10.4061/2011/457591

5. Weis JL (2019) Nontraumatic first rib fractures secondary to opposing muscle contractions. JBJS J Orthop Physician Assist 7:e0009. https://doi.org/10.2106/JBJS.JOPA.19.00009

6. Connolly LP, Connolly SA (2004) Rib stress fractures. Clin Nucl Med 29:614-616. https://doi.org/10.1097/00003072-20041 0000-00002

7. Findikoglu G, Yildiz BS, Sanlialp M et al (2015) Limitation of motion and shoulder disabilities in patients with cardiac implantable electronic devices. Int J Rehabil Res 38:287-293. https://doi. org/10.1097/MRR.0000000000000122

8. Akça AH, Şaşmaz Mİ, Kaplan Ş (2020) Kinesiotaping for isolated rib fractures in emergency department. Am J Emerg Med 38:638640. https://doi.org/10.1016/j.ajem.2019.11.049

Publisher's note Springer Nature remains neutral with regard to jurisdictional claims in published maps and institutional affiliations. 